\title{
Even Symmetric Parallel Linear Deterministic Interference Channels are Inseparable
}

\author{
Pritam Mukherjee $^{1}$, Ravi Tandon ${ }^{2}$, and Sennur Ulukus ${ }^{1}$ \\ ${ }^{1}$ Department of Electrical and Computer Engineering, University of Maryland College Park, MD 20742 \\ ${ }^{2}$ Department of Electrical and Computer Engineering, Virginia Tech, Blacksburg, VA 24061
}

\begin{abstract}
It is well known that parallel interference channels (ICs) are in general inseparable. Existing results illustrate the benefit of joint encoding across sub-channels by exploiting the asymmetry within the individual sub-channels. In this paper, we show, perhaps surprisingly, that symmetric parallel ICs are also inseparable. To this end, a class of parallel ICs is considered in which each individual sub-channel is a symmetric linear deterministic IC (LDIC). The capacity region of this class of parallel ICs is characterized. As a consequence, we find the necessary and sufficient conditions for inseparability of this class of parallel ICs.
\end{abstract}

\section{INTRODUCTION}

The interference channel (IC) is a canonical model to study the effect of interference in wireless networks. A long standing open problem in information theory, the IC was introduced by Shannon [1] and studied further by Ahlswede [2]. A complete characterization of the capacity region of the IC is still unknown today. The capacity region of the IC is known in the strong interference regime, where the interference at each receiver is stronger than the intended signal. Reference [3] showed that the single-user capacity for each user can be achieved when the interference is very strong. Reference [4] characterized the capacity region when the interference is strong. For the Gaussian IC, the capacity region is also known in the weak interference regime. References [5]-[7] showed that when the interference is sufficiently weak, the sum capacity is achieved by treating the interference as noise.

The best known achievable scheme for the IC is due to Han and Kobayashi [8], in which each transmitter splits its message into two parts, a common message which is decoded by both receivers and a private message that is decodable by only its intended receiver. A simplified version of the Han-Kobayashi scheme was used in [9] to characterize the capacity region of the Gaussian IC up to 1 bit. Reference [9] also introduced the concept of generalized degrees of freedom (g.d.o.f) and characterized the corresponding region for the two-user Gaussian IC. Reference [10] showed that the g.d.o.f. for the Gaussian IC has a one-to-one relationship with the capacity region of the corresponding linear deterministic IC (LDIC). The capacity region of the deterministic IC, found in [11], was used to characterize the capacity region of the two-user Gaussian IC up to a constant gap.

Besides the two-user Gaussian IC, the linear deterministic channel model has been successfully used as an intermediate step to approximate the capacity of other multi-terminal Gaussian networks. It was used in [12] to model the links in Gaussian networks with a single source-destination pair and an arbitrary number of relay nodes. Reference [12] showed that the capacity of the Gaussian network can be approximated by the capacity of the deterministic network up to a constant gap; 1 bit in special cases of single-relay and the two-relay Gaussian diamond network. In [13], the linear deterministic model was used to characterize the feedback capacity of ICs up to 2 bits. In [14], linear deterministic modeling was used to study the full-duplex bi-directional relay channel with two nodes and one relay. The proposed achievable scheme inspired by the deterministic model was shown to achieve rates within 3 bits of the capacity. The approximate sum capacity of the $K$-user symmetric Gaussian channel is found using deterministic modeling in [15].

Due to the usefulness of the linear deterministic model in approximating Gaussian channels accurately and simply, it has also been studied independently to reveal key insights relevant to Gaussian channels. Reference [16] studied the LDIC with partial feedback. In [17], the two-user LDIC is modeled as a game and the Nash equilibrium region is characterized. In reference [18], the combinatorial structure of the LDIC is explored to develop explicit deterministic coding schemes that achieve the sum capacity.

\section{A. Related Work}

The question of separability in a parallel Gaussian IC was raised by Cadambe and Jafar in [19]. They showed that the parallel Gaussian IC is not separable in general by presenting a specific example where joint coding outperforms individually optimal encoding. This came as a surprise since other well understood multi-terminal models such as the multiple access channel (MAC) and the broadcast channel (BC) are known to be separable, that is, the optimal transmission scheme for the Gaussian MAC or BC is independent encoding across the sub-channels with the final power allocation done through water-filling. The parallel Gaussian IC was studied in [20] with strong interference in every sub-channel, and achievable schemes based on independent encoding for each sub-channel were presented. Reference [21] studied the one-sided parallel Gaussian IC. By noting that the sum capacity is a concave function of power, a numerical algorithm was developed to compute the sum 
capacity. It was shown that a separation-based scheme with power allocation via water-filling performed close to optimal, when the interference is sufficiently weak. Reference [22] showed that independent encoding across sub-channels and treating the interference as noise is optimal if the interference in each sub-channel is sufficiently weak. Reference [23] studied an ergodic fading Gaussian IC with the channel state information known perfectly at each user. It was shown that in the case of uniformly strong interference (strong interference in every fading state), joint coding across the fading states (or sub-channels) is required for optimality. In the case of uniformly weak interference, however, independent coding for each fading state (or sub-channel) and treating interference as noise is optimal. In [24], the parallel LDIC with output feedback is studied using the combinatorial model developed in [18]. Reference [24] determined when the availability of output feedback can increase the sum capacity of parallel ICs.

Separability in parallel channels has important practical implications; it is much simpler to code for each sub-channel separately and optimize over just the power allocation than to perform joint encoding over all sub-channels. On the other hand, it is also important to understand how much can be benefitted (in terms of capacity) by joint encoding across sub-channels. In this paper, we address this very question of separability. As a stepping stone to understand the parallel Gaussian IC, in this paper, we focus on the twouser parallel LDICs with two symmetric sub-channels. We establish the capacity region of such channels completely, which enables us to address the question of separability directly. Interestingly, we show that separability with respect to the sum capacity is equivalent to separability with respect to the full capacity region.

\section{System MODEL}

A two-user parallel LDIC is considered. The parallel channel comprises of two sub-channels, each one of which is a symmetric LDIC. In this setting, there are two transmitterreceiver pairs: $\mathrm{Tx}_{1}$ (respectively $\mathrm{Tx}_{2}$ ) wishes to send a message $W_{1}$ (respectively $W_{2}$ ) to $\mathrm{Rx}_{1}$ (respectively $\mathbf{R x}_{2}$ ) through the two parallel sub-channels. A symmetric subchannel, where the number of direct signal levels at $\mathrm{Rx}_{1}$ is the same as that for $\mathrm{Rx}_{2}$, and the number of interference levels at $\mathrm{Rx}_{1}$ is the same as that for $\mathrm{Rx}_{2}$, is fully described by two parameters: $(m, n)$, where $n$ denotes the number of direct signal levels from $\mathrm{Tx}_{i}$ to $\mathrm{Rx}_{i}$, for $i=1,2$ and $m$ denotes the number of cross or interfering signal levels from $\mathrm{Tx}_{i}$ to $\mathrm{Rx}_{j}$, for $i \neq j$ and $i, j=1,2$. We denote the two sub-channels by sub-channel $A$ and sub-channel $B$, with parameters $\left(m_{A}, n_{A}\right)$ and $\left(m_{B}, n_{B}\right)$. Thus, we collectively refer to the overall channel by four parameters: the $\left(m_{A}, n_{A}, m_{B}, n_{B}\right)$-LDIC. Fig. 1 shows the $(1,2,2,1)$ LDIC with $m_{A}=1, n_{A}=2, m_{B}=2, n_{B}=1$. In addition to the four parameters $\left(m_{A}, n_{A}, m_{B}, n_{B}\right)$, we find it useful to define the following two parameters:

$$
\alpha_{A} \triangleq \frac{m_{A}}{n_{A}}, \quad \alpha_{B} \triangleq \frac{m_{B}}{n_{B}}
$$
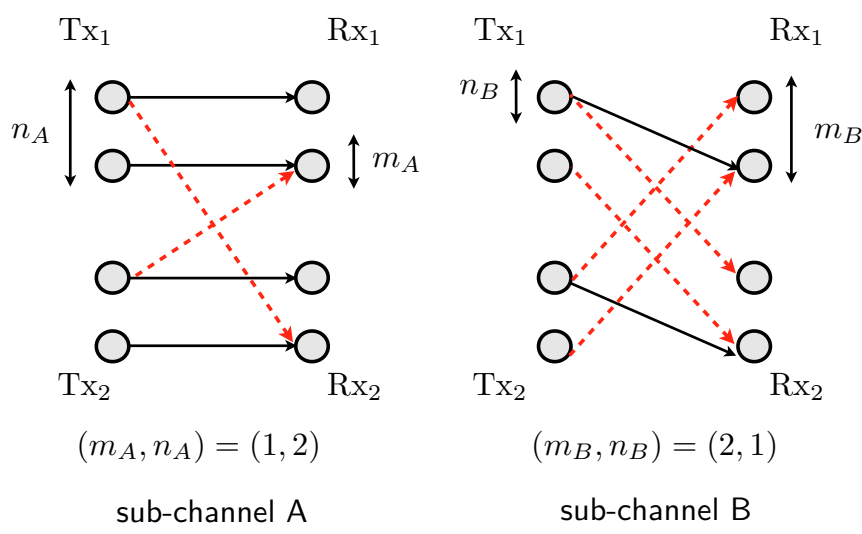

Fig. 1: The (1, 2, 2, 1)-LDIC.

which measure the strength of interference relative to the direct signals for each of the individual sub-channels.

\section{Main Results AND Discussion}

Theorem 1 The capacity region of the parallel LDIC described by $\left(m_{A}, n_{A}, m_{B}, n_{B}\right)$ is given by:

$$
\begin{aligned}
R_{1} \leq n_{A} & +n_{B} \\
R_{2} \leq n_{A} & +n_{B} \\
R_{1} & +R_{2} \leq \max \left(m_{A}, 2 n_{A}-m_{A}\right)+\max \left(m_{B}, 2 n_{B}-m_{B}\right) \\
R_{1}+R_{2} \leq & 2\left[\max \left(m_{A}, n_{A}-m_{A}\right)+\max \left(m_{B}, n_{B}-m_{B}\right)\right] \\
2 R_{1} & +R_{2} \leq \max \left(m_{A}, 2 n_{A}-m_{A}\right)+\max \left(m_{A}, n_{A}-m_{A}\right) \\
& \quad+\max \left(m_{B}, 2 n_{B}-m_{B}\right)+\max \left(m_{B}, n_{B}-m_{B}\right) \\
R_{1}+2 R_{2} & \leq \max \left(m_{A}, 2 n_{A}-m_{A}\right)+\max \left(m_{A}, n_{A}-m_{A}\right) \\
& \quad+\max \left(m_{B}, 2 n_{B}-m_{B}\right)+\max \left(m_{B}, n_{B}-m_{B}\right)
\end{aligned}
$$

We next present the rate region that can be achieved by separate encoding across the two sub-channels [10]:

$$
\begin{aligned}
& R_{1} \leq n_{A}+n_{B} \\
& R_{2} \leq n_{A}+n_{B} \\
& R_{1}+R_{2} \leq \min \left[\max \left(2 n_{A}-m_{A}, m_{A}\right),\right. \\
& \left.\quad 2 \max \left(m_{A}, n_{A}-m_{A}\right)\right]+\min \left[\max \left(2 n_{B}-m_{B}, m_{B}\right),\right. \\
& \left.\quad 2 \max \left(m_{B}, n_{B}-m_{B}\right)\right] \\
& 2 R_{1}+R_{2} \leq \max \left(2 n_{A}-m_{A}, m_{A}\right)+\max \left(m_{A}, n_{A}-m_{A}\right) \\
& \quad+\max \left(2 n_{B}-m_{B}, m_{B}\right)+\max \left(m_{B}, n_{B}-m_{B}\right) \\
& R_{1}+2 R_{2} \leq \max \left(2 n_{A}-m_{A}, m_{A}\right)+\max \left(m_{A}, n_{A}-m_{A}\right) \\
& \quad+\max \left(2 n_{B}-m_{B}, m_{B}\right)+\max \left(m_{B}, n_{B}-m_{B}\right)
\end{aligned}
$$

In the following sub-section, we give a motivating example, in which we compare these two regions and show that separation is strictly sub-optimal, i.e., that the capacity region can strictly exceed the region achievable by separately optimal encoding over parallel channels.

\section{A. Sub-Optimality of Separation: A Motivating Example}

Let us now consider the $(1,2,2,1)$-LDIC introduced in the previous section and shown in Fig. 1. For this parallel 


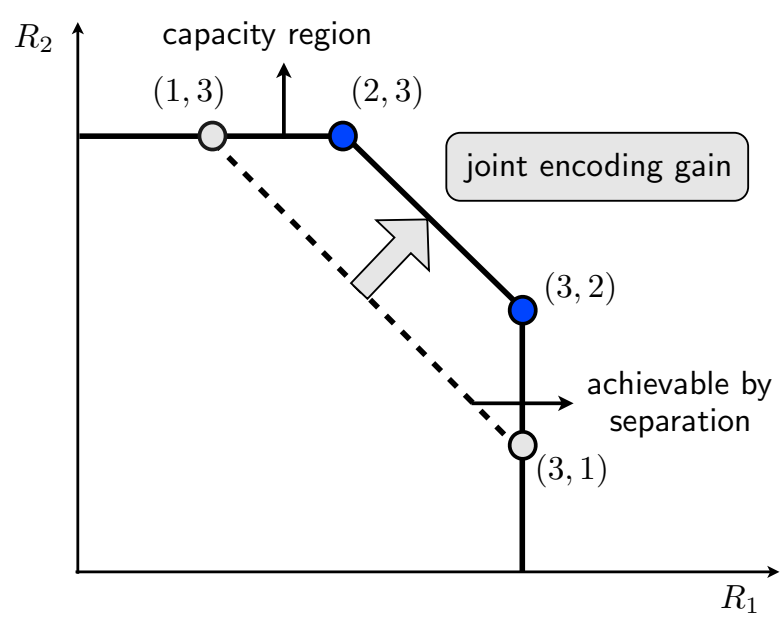

Fig. 2: Capacity region for the $(1,2,2,1)$-LDIC.

LDIC, the capacity region from Theorem 1 is given by

$$
\begin{array}{r}
R_{1} \leq 3 \\
R_{2} \leq 3 \\
R_{1}+R_{2} \leq 5
\end{array}
$$

On the other hand, the region achieved by separate encoding across the two sub-channels is given by

$$
\begin{array}{r}
R_{1} \leq 3 \\
R_{2} \leq 3 \\
R_{1}+R_{2} \leq 4
\end{array}
$$

Fig. 2 shows that independent encoding across sub-channels is a strictly suboptimal strategy in this case.

The optimal encoding that uses both the sub-channels jointly is shown in Fig. 3 along with the separation based scheme. Here, we show that with separate encoding we can achieve the rate pair $(3,1)$, whereas by joint encoding across sub-channels, we can achieve the rate pair $(3,2)$. The key idea in joint encoding is the efficient utilization of signal levels at the receivers that are left empty in the separation based scheme. In particular, in the separation based scheme, in sub-channel $A$, the top-most level of receiver 2 is unused. By carefully designing joint encoding schemes, one can make full use of such under-utilized signal levels, thereby improving upon separation.

As a consequence of Theorem 1, we have the following proposition:

Proposition 1 The $\left(m_{A}, n_{A}, m_{B}, n_{B}\right)$-LDIC is separable if and only if the pair $\left(\alpha_{A}, \alpha_{B}\right)$ falls into one of the following three regions (see also Fig. 4):

- $\alpha_{A} \in\left[0, \frac{2}{3}\right], \alpha_{B} \in\left[0, \frac{2}{3}\right]$.

- $\alpha_{A} \in\left[\frac{2}{3}, 2\right], \alpha_{B} \in\left[\frac{2}{3}, 2\right]$.

- $\alpha_{A} \geq 2, \alpha_{B} \geq 2$.

For all the remaining cases, the separation based scheme is strictly sub-optimal.

We note that the capacity region in Theorem 1 and the
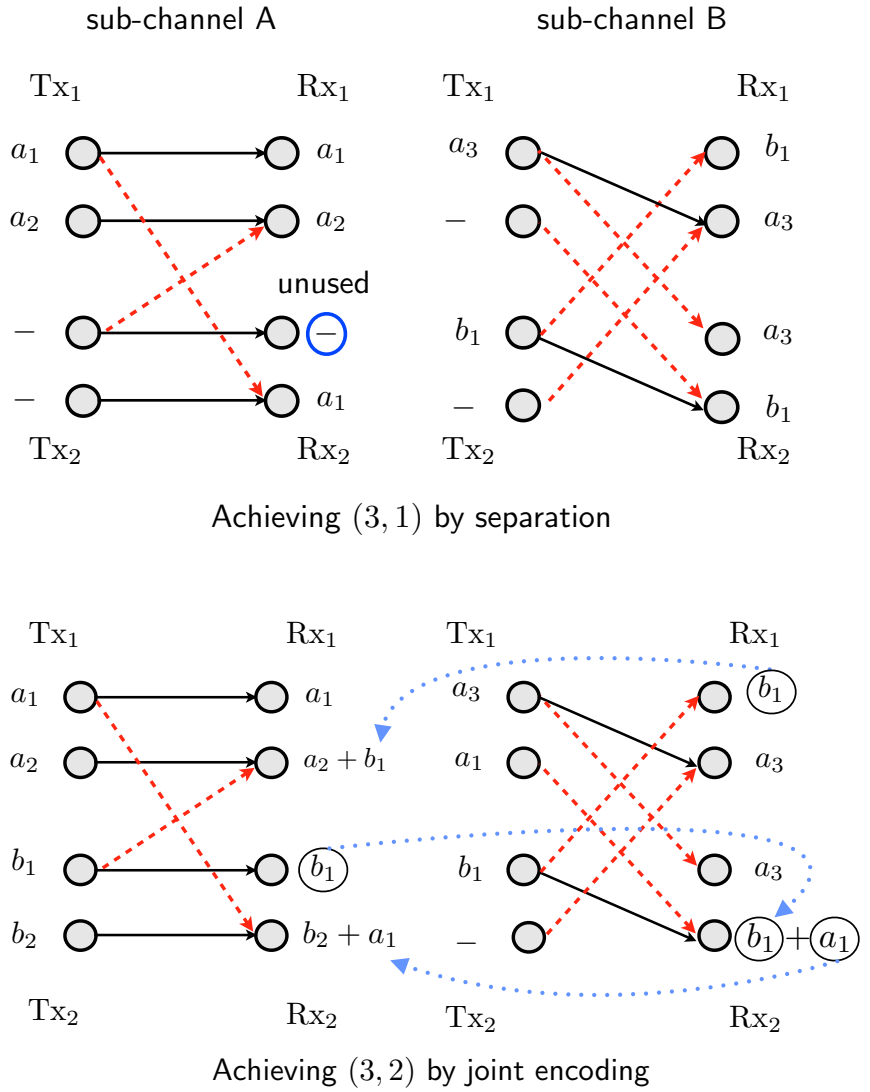

Fig. 3: Illustration of joint encoding versus separation.

separation based rate region only differ in $R_{1}+R_{2}$ bounds. Hence, the proposition directly follows by comparing the sum rate bounds. As this is rather straightforward, we skip this analysis, and instead, highlight some interesting consequences and insights emerging from this result.

\section{B. Remarks}

1) It is perhaps surprising that even though the individual sub-channels are symmetric, the parallel LDIC may still be inseparable. In almost all examples of inseparability we have previously encountered in the literature, e.g., [19], the two sub-channels were not symmetric individually. To the best of our knowledge, we have presented here the first examples of inseparability even when the individual sub-channels are symmetric.

2) It is easy to verify that separability with respect to the sum rate is equivalent to separability with respect to the capacity region.

3) Recall from Theorem 1 that there are two sum rate bounds in the capacity region of a symmetric LDIC. For a given value of the parameter $\alpha$, only one of the two bounds is active. If we examine Fig. 4, and keep in mind the previous remark, we can infer that the parallel LDIC is separable if and only if the same sum rate constraint is active for both sub-channels. This observation may be useful to generalize our results for parallel LDICs with more than two sub-channels. 


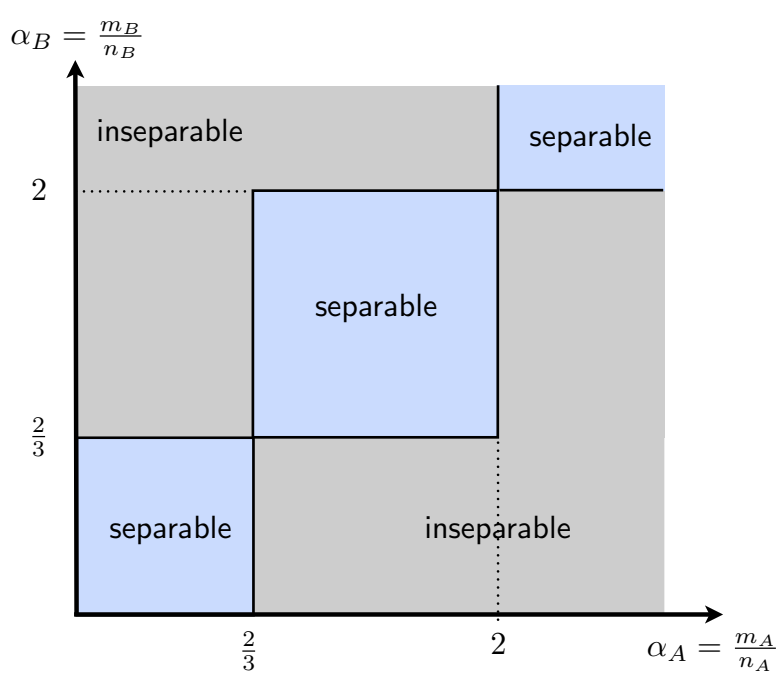

Fig. 4: Separable versus inseparable regions.

\section{Proof of Theorem 1}

\section{A. Converse}

We note here that the parallel LDIC considered in this paper falls in the class of deterministic ICs studied by Costa and El Gamal in [11]. In the Costa-El Gamal model, the input output relationships are given as follows:

$$
Y_{1}=f_{1}\left(X_{1}, V_{2}\right), \quad Y_{2}=f_{2}\left(X_{2}, V_{1}\right)
$$

where $X_{1}$ and $X_{2}$ are the channel inputs, the interference $V_{1}=g_{1}\left(X_{1}\right)$ at receiver 2 is a function of $X_{1}$, the interference $V_{2}=g_{2}\left(X_{2}\right)$ at receiver 1 is a function of $X_{2}$. In addition, the following conditions also hold:

$$
H\left(Y_{1} \mid X_{1}\right)=H\left(V_{2}\right), \quad H\left(Y_{2} \mid X_{2}\right)=H\left(V_{1}\right)
$$

For this class of deterministic ICs satisfying (9), [11] characterized the capacity region, which is given by

$$
\begin{aligned}
R_{1} & \leq H\left(Y_{1} \mid V_{2}\right) \\
R_{2} & \leq H\left(Y_{2} \mid V_{1}\right) \\
R_{1}+R_{2} & \leq H\left(Y_{1} \mid V_{1} V_{2}\right)+H\left(Y_{2}\right) \\
R_{1}+R_{2} & \leq H\left(Y_{1}\right)+H\left(Y_{2} \mid V_{1} V_{2}\right) \\
R_{1}+R_{2} & \leq H\left(Y_{1} \mid V_{1}\right)+H\left(Y_{2} \mid V_{2}\right) \\
2 R_{1}+R_{2} & \leq H\left(Y_{1}\right)+H\left(Y_{1} \mid V_{1}, V_{2}\right)+H\left(Y_{2} \mid V_{2}\right) \\
R_{1}+2 R_{2} & \leq H\left(Y_{2}\right)+H\left(Y_{2} \mid V_{1}, V_{2}\right)+H\left(Y_{1} \mid V_{1}\right)
\end{aligned}
$$

We next note that the parallel LDIC considered in this paper falls in the class of Costa El-Gamal deterministic IC. To note this, we denote by vectors $\mathbf{X}_{1 A}$ and $\mathbf{X}_{1 B}$ the inputs to the channel from $\mathrm{Tx}_{1}$ in the sub-channels $A$ and $B$, respectively. We define $\mathbf{X}_{2 A}$ and $\mathbf{X}_{2 B}$ similarly. The output at $\mathrm{Rx}_{1}$ in sub-channel $A$ and $B$ are denoted by $\mathbf{Y}_{1 A}$ and $\mathbf{Y}_{1 B}$, respectively. The interference at $\mathrm{Rx}_{2}$ due to $\mathrm{Tx}_{1}$ in sub-channels $A$ and $B$ are denoted by $\mathbf{V}_{1 A}$ and $\mathbf{V}_{1 B}$, respectively. Similarly for $\mathbf{V}_{2 A}$ and $\mathbf{V}_{2 B}$. Let $X_{1}=\left(\mathbf{X}_{1 A}, \mathbf{X}_{1 B}\right)$. Similarly, define $X_{2}, Y_{1}, Y_{2}, V_{1}$ and $V_{2}$. Now we note that $V_{1}$ and $V_{2}$ are deterministic functions of $X_{1}$ and $X_{2}$, respectively. Also, $Y_{1}$ is determined completely by $X_{1}$ and $V_{2}$. A similar statement holds for $Y_{2}$. Finally, it is straightforward to check that with these definitions the conditions in (9) hold. The converse now can be obtained using (10)-(16). An observation similar to [10, Lemma 4], that the optimal input distribution is uniform, allows us to simplify the rate region in [11] further by noting that

$$
\begin{aligned}
H\left(Y_{1} \mid V_{2}\right) & \leq H\left(\mathbf{Y}_{1 A} \mid \mathbf{V}_{2 A}\right)+H\left(\mathbf{Y}_{1 B} \mid \mathbf{V}_{2 B}\right) \\
& \leq n_{A}+n_{B} \\
H\left(Y_{1}\right) & \leq H\left(\mathbf{Y}_{1 A}\right)+H\left(\mathbf{Y}_{1 B}\right) \\
& \leq \max \left(m_{A}, n_{A}\right)+\max \left(m_{B}, n_{B}\right) \\
H\left(Y_{1} \mid V_{1}, V_{2}\right) & \leq H\left(\mathbf{Y}_{1 A} \mid \mathbf{V}_{1 A}, \mathbf{V}_{2 A}\right)+H\left(\mathbf{Y}_{1 B} \mid \mathbf{V}_{1 B}, \mathbf{V}_{2 B}\right) \\
& \leq \max \left(n_{A}-m_{A}, 0\right)+\max \left(n_{B}-m_{B}, 0\right) \\
H\left(Y_{1} \mid V_{1}\right) & \leq H\left(\mathbf{Y}_{1 A} \mid \mathbf{V}_{1 A}\right)+H\left(\mathbf{Y}_{1 B} \mid \mathbf{V}_{1 B}\right) \\
& \leq \max \left(m_{A}, n_{A}-m_{A}\right)+\max \left(m_{B}, n_{B}-m_{B}\right)
\end{aligned}
$$

Using symmetry within each of the sub-channels $A$ and $B$ and the above equations to simplify the rate region in (10)(16) gives us the rate region specified in Theorem 1. We note that unlike in [11], we have two rather than three sum rate bounds; this follows due the assumed symmetry for each of the two sub-channels in our case.

\section{B. Achievability}

We first note that the Han-Kobayashi scheme [8] achieves the capacity of Costa-El Gamal model and hence also for our parallel LDIC model. However, this scheme is based on random coding arguments and moreover the vector (multichannel) version of it permits arbitrary ways of encoding and decoding information across sub-channels. Thus, the structure of how to jointly encode across sub-channels is not transparent. Furthermore, we are ultimately interested in taking the insights from the linear to the Gaussian model. It is therefore desirable to seek explicit capacity achieving schemes which clearly highlight how to jointly encode across sub-channels for the parallel LDIC. This is one of the main contributions of this work and the focus of this section.

We already know that the separation based scheme achieves the capacity in three $\left(\alpha_{A}, \alpha_{B}\right)$ regimes as stated in Proposition 1. Hence, to prove the achievability, we only need to consider the $\left(\alpha_{A}, \alpha_{B}\right)$ regimes in which separation is sub-optimal. In addition, due to symmetry, it suffices to consider $\alpha_{B} \geq \alpha_{A}$. Hence, the regimes that we need to consider are the following:

- Regime 1: $\alpha_{A} \in\left[0, \frac{2}{3}\right], \alpha_{B} \geq \frac{2}{3}$.

- Regime 2: $\alpha_{A} \in\left[\frac{2}{3}, 2\right], \alpha_{B} \geq 2$.

Due to space limitations here, we restrict ourselves to one sub-case of Regime 1 in which $\alpha_{A} \in[0,2 / 3]$ and $\alpha_{B} \leq 1$, and one sub-case of Regime 2, in which $\alpha_{A} \geq 1$. All of the remaining cases will be presented in a longer version of this paper. Hence, we sub-divide the regimes to be considered in this paper in detail as follows:

- Regime $1 a: \alpha_{A} \in\left[0, \frac{1}{2}\right], \alpha_{B} \in\left[\frac{2}{3}, 1\right]$.

- Regime $1 b: \alpha_{A} \in\left[\frac{1}{2}, \frac{2}{3}\right], \alpha_{B}\left[\frac{2}{3}, 1\right]$.

- Regime $2 a: \alpha_{A} \in[1,2], \alpha_{B} \geq 2$. 

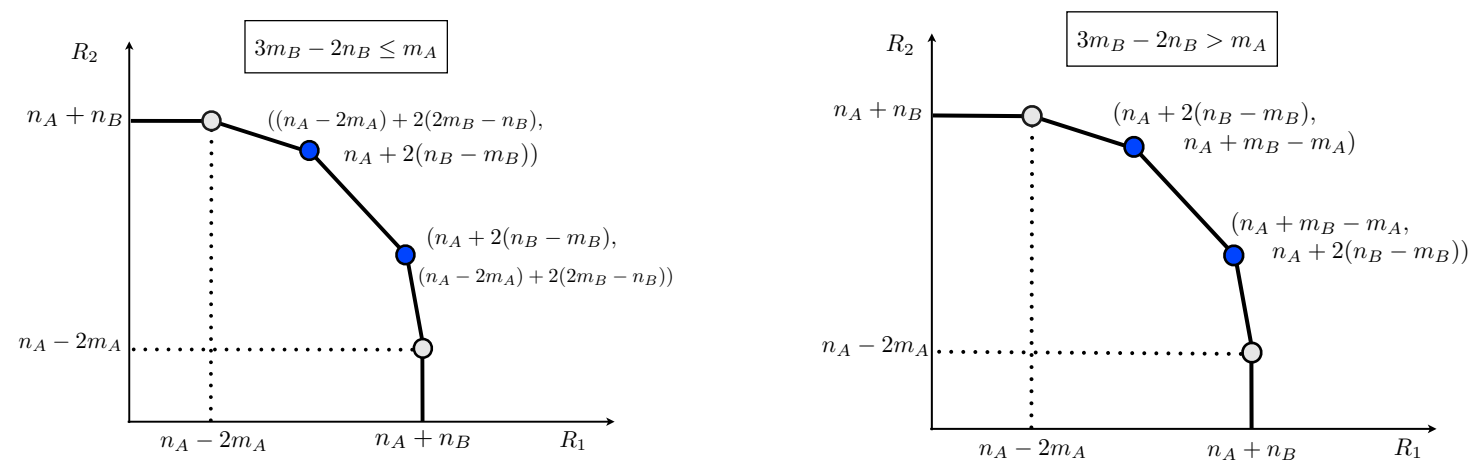

Fig. 5: Capacity region for Regime $1 a$.
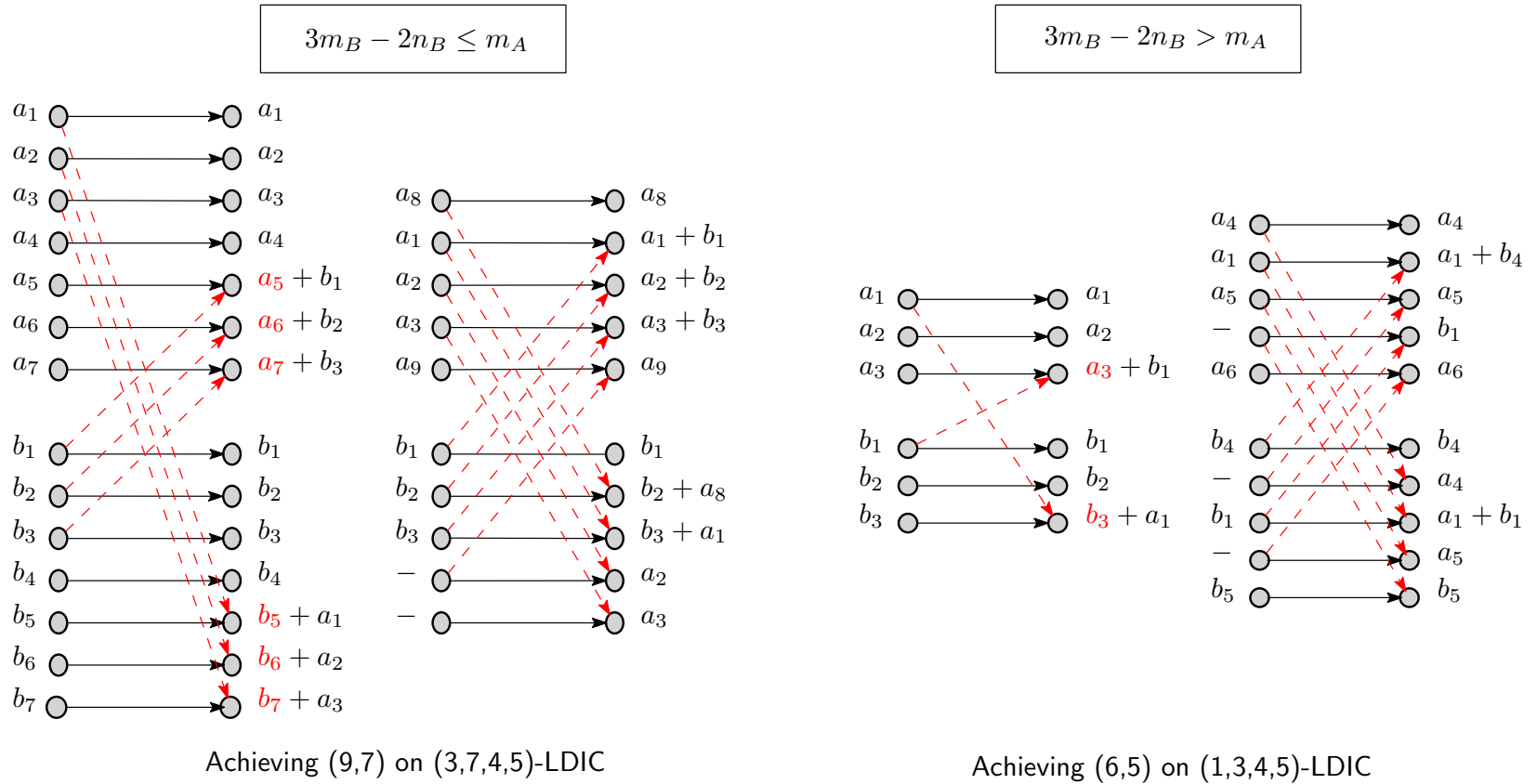

Fig. 6: Representative examples for Regime $1 a$.

We can think of Regime $1 a$ as a combination of very weak and moderate interference sub-channels. Similarly, Regime $1 b$ can be regarded as a combination of weak and moderate interference sub-channels, and Regime $2 a$ can be regarded as a combination of strong and very strong interference sub-channels.

\section{Achievability: Regime $1 a$}

In this regime, the region in Theorem 1 simplifies to:

$$
\begin{aligned}
R_{1} & \leq n_{A}+n_{B} \\
R_{2} & \leq n_{A}+n_{B} \\
R_{1}+R_{2} & \leq\left(2 n_{A}-m_{A}\right)+\left(2 n_{B}-m_{B}\right) \\
R_{1}+R_{2} & \leq 2\left(n_{A}-m_{A}\right)+2 m_{B} \\
2 R_{1}+R_{2} & \leq 3 n_{A}-2 m_{A}+2 n_{B} \\
R_{1}+2 R_{2} & \leq 3 n_{A}-2 m_{A}+2 n_{B}
\end{aligned}
$$

We next note that if $3 m_{B}-2 n_{B} \leq m_{A}$, the constraint in (19) is inactive whereas (20) is active. On the other hand, for $3 m_{B}-2 n_{B}>m_{A}$, the opposite is true. These two sub-divisions of Regime $1 a$ are shown in Fig. 5. For both of these cases, the achievability of $\left(n_{A}+n_{B}, n_{A}-2 m_{A}\right)$ follows directly by using a separation based scheme. To note this, we observe that in sub-channel $A$, we can achieve the rate pair $\left(n_{A}, n_{A}-2 m_{A}\right)$. For sub-channel $B$, the rate pair $\left(n_{B}, 0\right)$ is achievable. Hence, the rate pair $\left(n_{A}+n_{B}, n_{A}-2 m_{A}\right)$ is achievable for the parallel LDIC. We therefore focus on the non-trivial points on the capacity region.

1) $3 m_{B}-2 n_{B} \leq m_{A}$ : Here, we focus on achieving the following pair:

$$
\begin{aligned}
& R_{1}=n_{A}+2\left(n_{B}-m_{B}\right) \\
& R_{2}=\left(n_{A}-2 m_{A}\right)+2\left(2 m_{B}-n_{B}\right)
\end{aligned}
$$

To achieve this rate pair, $\mathrm{Tx}_{1}$ transmits $n_{A}$ symbols in subchannel $A$, and $\left(n_{B}-m_{B}\right)$ symbols from the top and $\left(n_{B}-\right.$ $\left.m_{B}\right)$ symbols from the bottom in sub-channel $B$. If $\left(2 m_{B}-\right.$ $\left.n_{B}\right) \leq m_{A}, \mathrm{Tx}_{2}$ transmits $\left(2 m_{B}-n_{B}\right)$ symbols from the top and $\left(2 m_{B}-n_{B}\right)$ symbols from the bottom and $\left(n_{A}-2 m_{A}\right)$ symbols from the middle levels. The interference at each user is $\left(2 m_{B}-n_{B}\right)$, which can be resolved in sub-channel $B$. If $\left(2 m_{B}-n_{B}\right) \geq m_{A}, \mathrm{Tx}_{2}$ sends $n_{A}$ symbols in subchannel $A$, and $\left(2 m_{B}-n_{B}-m_{A}\right)$ symbols from the top and 

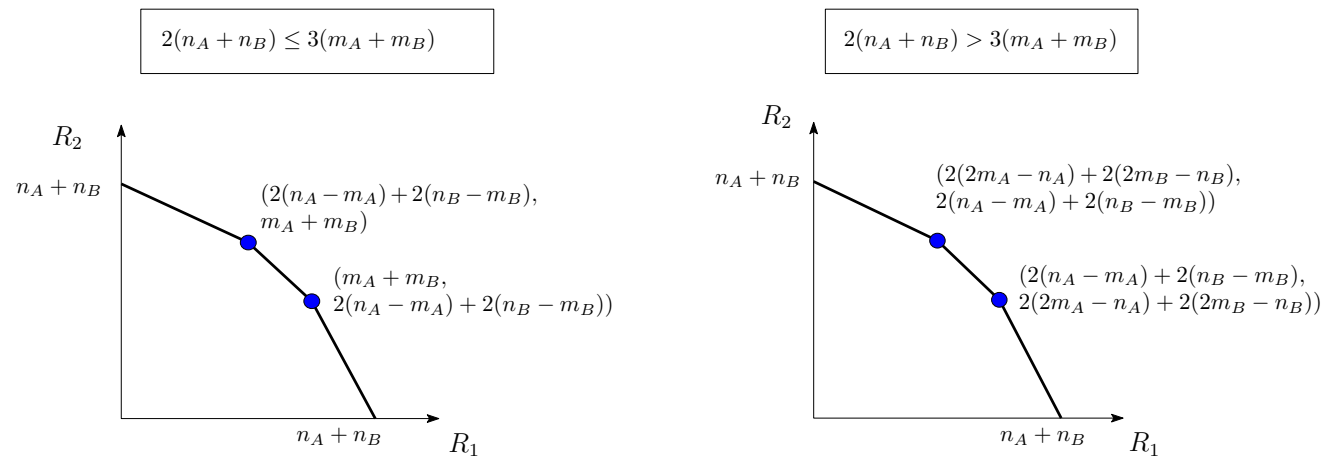

Fig. 7: Capacity region for Regime $1 b$.

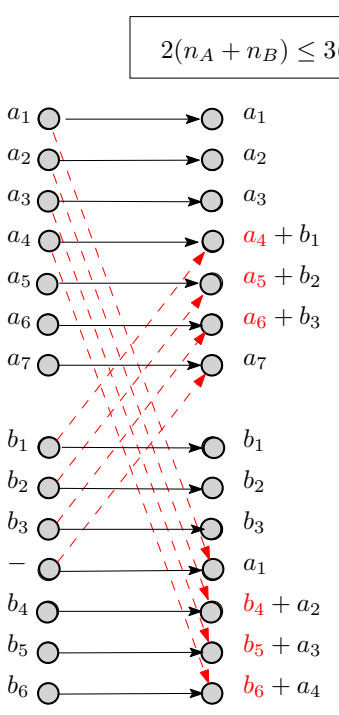

Achieving $(9,8)$ on the $(4,7,5,6)$-LDIC

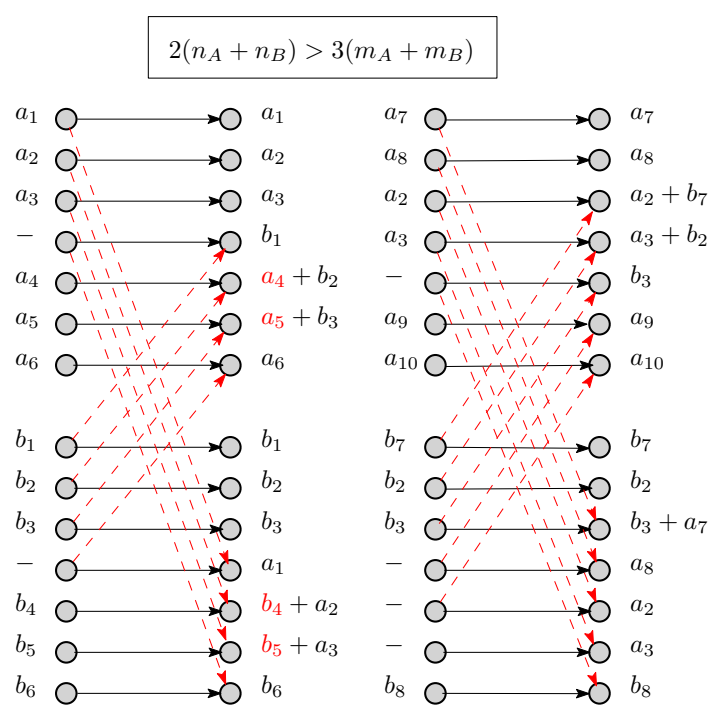

Achieving $(10,8)$ on the $(4,7,5,7)$-LDIC

Fig. 8: Representative examples for Regime $1 b$.

$\left(2 m_{B}-n_{B}-m_{A}\right)$ symbols from the bottom levels in subchannel $B$. The interference of $m_{A}$ symbols in sub-channel $A$ can be resolved in sub-channel $B$.

2) $3 m_{B}-2 n_{B}>m_{A}$ : Here, we focus on achieving the following pair:

$$
\begin{aligned}
& R_{1}=n_{A}+\left(m_{B}-m_{A}\right) \\
& R_{2}=n_{A}+2\left(n_{B}-m_{B}\right)
\end{aligned}
$$

To achieve this rate pair, $\mathrm{Tx}_{1}$ and $\mathrm{Tx}_{2}$ transmit $n_{A}$ symbols in sub-channel $A$ causing interference of $m_{A}$ symbols at each receiver. In sub-channel $B, \mathrm{Tx}_{1}$ and $\mathrm{Tx}_{2}$ transmit on the top and bottom $\left(n_{B}-m_{B}\right)$ levels. Additionally, $\mathrm{Tx}_{1}$ transmits $\left(m_{B}-m_{A}\right)-2\left(n_{B}-m_{B}\right)$ new symbols beneath the top $2\left(n_{B}-m_{B}\right)$ levels. The remaining levels for sub-channel $B$ can be used to mitigate the interference in sub-channel $A$.

As illustrative examples in Regime $1 a$, we consider two LDICs: the (3,7,4,5)-LDIC and the (1,3,4,5)-LDIC. Both of these LDICs belong to Regime $1 a$. For the $(3,7,4,5)$-LDIC, $3 m_{B}-2 n_{B}=2 \leq m_{A}=3$, while for the $(1,3,4,5)$ LDIC, the opposite inequality is true. The non-trivial points to achieve are $(9,7)$ for the $(3,7,4,5)$-LDIC and $(6,5)$ for the $(1,3,4,5)$-LDIC. We show how these points can be achieved in Fig. 6. Note how joint encoding over the two sub-channels has been used to achieve these points; the interference in sub-channel $A$ is mitigated in sub-channel $B$.

\section{Achievability: Regime $1 b$}

In this regime, the region in Theorem 1 simplifies to:

$$
\begin{aligned}
R_{1} & \leq n_{A}+n_{B} \\
R_{2} & \leq n_{A}+n_{B} \\
R_{1}+R_{2} & \leq\left(2 n_{A}-m_{A}\right)+\left(2 n_{B}-m_{B}\right) \\
R_{1}+R_{2} & \leq 2 m_{A}+2 m_{B} \\
2 R_{1}+R_{2} & \leq 2 n_{A}+2 n_{B} \\
R_{1}+2 R_{2} & \leq 2 n_{A}+2 n_{B}
\end{aligned}
$$

We note that if $2\left(n_{A}+n_{B}\right) \leq 3\left(m_{A}+m_{B}\right)$, (30) is redundant and only (29) is active. Fig. 7 shows the capacity regions in these two cases. Let us focus on the two cases separately.

1) $2\left(n_{A}+n_{B}\right) \leq 3\left(m_{A}+m_{B}\right)$ : Here we must achieve the following rate pair:

$$
\begin{aligned}
& R_{1}=m_{A}+m_{B} \\
& R_{2}=2\left(n_{A}-m_{A}\right)+2\left(n_{B}-m_{B}\right)
\end{aligned}
$$



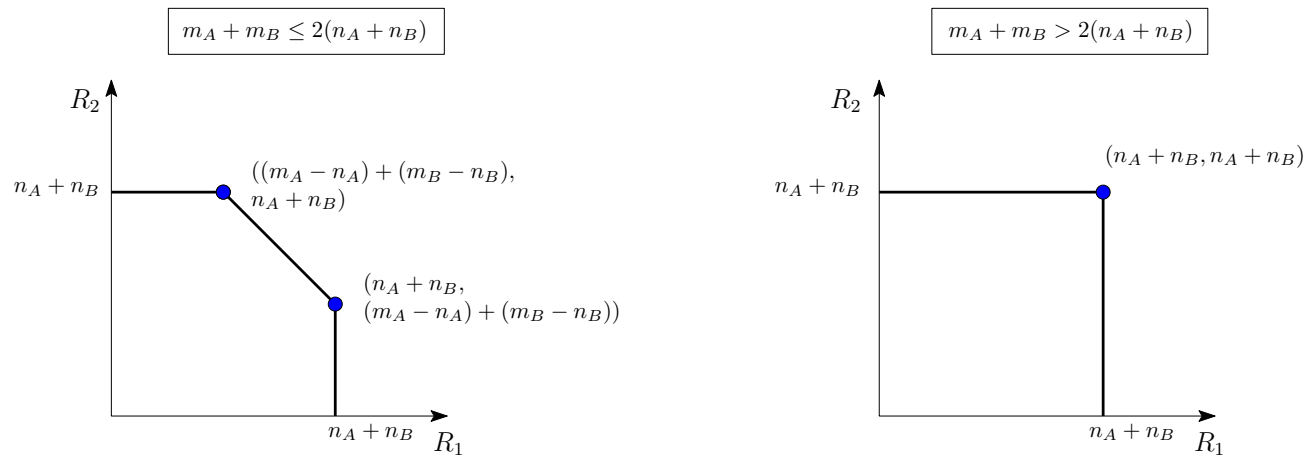

Fig. 9: Capacity region of Regime $2 a$.

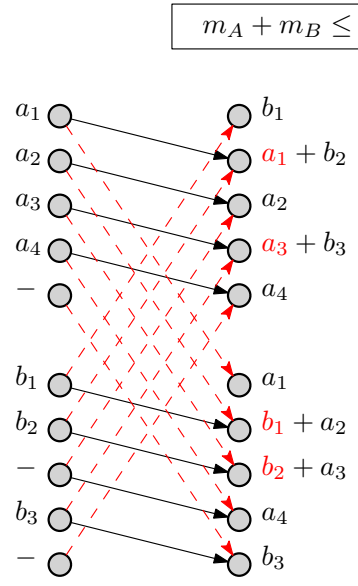

Achieving $(5,3)$ on the $(5,4,3,1)$-LDIC

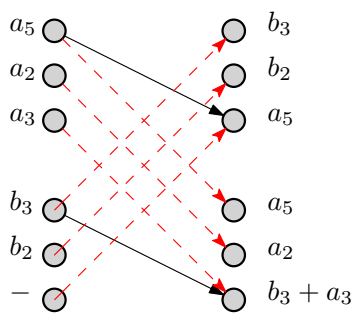

Fig. 10: Representative examples for Regime $2 a$.
We identify two subcases:

Case a: $m_{A}+m_{B} \geq n_{A}$ : $\mathrm{Tx}_{1}$ sends $n_{A}$ symbols in subchannel $A$ and the remaining symbols in sub-channel $B$. $\mathrm{Tx}_{2}$ sends $\left(n_{A}-m_{A}\right)$ symbols from the top and the bottom in sub-channel $A$ and $\left(n_{B}-m_{B}\right)$ symbols from the top and the bottom in sub-channel $B$. The interference in sub-channel $A$ is $\left(n_{A}-m_{A}\right)$, which can be resolved in sub-channel $B$.

Case b: $m_{A}+m_{B}<n_{A}$ : $\mathrm{Tx}_{1}$ transmits $\left(3 m_{B}-2 n_{B}\right)+$ $\left(2 m_{A}-n_{A}\right)$ symbols from the top and $\left(n_{A}-m_{A}\right)$ symbols from the bottom in sub-channel $A$. $\mathrm{Tx}_{2}$ transmits $\left(n_{A}-\right.$ $m_{A}$ ) symbols from the top and the bottom in sub-channel $A$ and $\left(n_{B}-m_{B}\right)$ symbols from the top and the bottom in sub-channel $B$. The interference in sub-channel $A$ at $\mathrm{Rx}_{1}$ and $\mathrm{Rx} 2$ is $\left(3 m_{B}-2 n_{B}\right)$ symbols. In sub-channel $B, \mathrm{Tx}_{1}$ transmits $\left(n_{B}-m_{B}\right)$ remaining symbols from the top and the bottom, stays silent in $\left(n_{B}-m_{B}\right)$ levels to avoid interference at $\mathrm{Rx}_{2}$ and transmits $\left(3 m_{B}-2 n_{B}\right)$ symbols in $\left(3 m_{B}-\right.$ $2 n_{B}$ ) levels remaining among the top $m_{B}$ levels. Thus, the interference is resolved in sub-channel $B$.

2) $2\left(n_{A}+n_{B}\right)>3\left(m_{A}+m_{B}\right):$ In this case, we need to achieve the following rate pair:

$$
\begin{aligned}
& R_{1}=2\left(n_{A}-m_{A}\right)+2\left(n_{B}-m_{B}\right) \\
& R_{2}=2\left(2 m_{A}-n_{A}\right)+2\left(2 m_{B}-n_{B}\right)
\end{aligned}
$$

$\mathrm{Tx}_{1}$ and $\mathrm{Tx}_{2}$ each send $\left(n_{A}-m_{A}\right)$ symbols from the top and the bottom in sub-channel $A$, causing an interference of $\left(2 n_{A}-3 m_{A}\right)$ symbols at each user. In sub-channel $B, \mathrm{Tx}_{1}$ transmits $\left(n_{B}-m_{B}\right)$ symbols from the top and the bottom, while $\mathrm{Tx}_{2}$ sends $\left(2 m_{B}-n_{B}\right)-\left(2 n_{A}-3 m_{A}\right)$ symbols from the top and the bottom. The interference is resolved in subchannel $B$.

As illustrative examples in Regime $1 b$, we consider two LDICs: the $(4,7,5,6)$-LDIC and the $(4,7,5,7)$-LDIC. For the $(4,7,5,6)$-LDIC, we have $2\left(n_{A}+n_{B}\right)=26 \leq 3\left(m_{A}+m_{B}\right)=$ 27. We are interested in achieving the rate pair $(9,8)$ on this LDIC. For the $(4,7,5,7)$-LDIC, $2\left(n_{A}+n_{B}\right)=28>3\left(m_{A}+\right.$ $\left.m_{B}\right)=27$. Thus, we are interested in achieving the rate pair $(10,8)$. Fig. 8 shows how the desired rate pairs are achieved on the corresponding LDICs. Again, joint encoding on the two sub-channels is exploited.

\section{E. Achievability: Regime $2 a$}

In this regime, the region in Theorem 1 simplifies to:

$$
\begin{aligned}
R_{1} & \leq n_{A}+n_{B} \\
R_{2} & \leq n_{A}+n_{B} \\
R_{1}+R_{2} & \leq m_{A}+m_{B}
\end{aligned}
$$

We note that if $m_{A}+m_{B}>2\left(n_{A}+n_{B}\right)$, the sum rate bound is inactive. Fig. 9 shows the capacity region in each case. Let 
us now focus on the two cases:

1) $m_{A}+m_{B} \leq 2\left(n_{A}+n_{B}\right):$ In this case, we are interested in achieving the rate pair:

$$
\begin{aligned}
& R_{1}=n_{A}+n_{B} \\
& R_{2}=\left(m_{A}-n_{A}\right)+\left(m_{B}-n_{B}\right)
\end{aligned}
$$

We identify several subcases:

Case $a:\left(m_{A}-n_{A}\right)+\left(m_{B}-n_{B}\right) \geq n_{A}$ : To achieve the desired rate pair, $\mathrm{Tx}_{1}$ sends $\left(n_{A}+n_{B}\right)$ symbols using subchannels $A$ and $B$. Tx ${ }_{2}$ sends $n_{A}$ symbols in sub-channel $A$ and the remaining $\left(m_{A}-2 n_{A}+m_{B}-n_{B}\right)$ symbols from top levels in sub-channel $B$. The interference of $2 n_{A}-m_{A}$ symbols in sub-channel $A$ can be resolved in sub-channel $B$.

Case b: $\left(m_{A}-n_{A}\right)+\left(m_{B}-n_{B}\right) \leq n_{A}$ and $m_{B}-$ $n_{B} \geq m_{A}-n_{A}$ : In this case, $\mathrm{Tx}_{1}$ sends $\left(n_{A}+n_{B}\right)$ symbols using sub-channels $A$ and $B$ as usual. Tx $x_{2}$ sends $\left(m_{B}-n_{B}\right)$ symbols from the top and the remaining $\left(m_{A}-n_{A}\right)$ symbols right underneath the top $\left(2 n_{A}-m_{A}\right)$ levels in sub-channel $A$. The interference at each receiver is $\left(m_{B}-n_{B}\right)$ symbols which can be resolved in sub-channel $B$.

Case c: $\left(m_{A}-n_{A}\right)+\left(m_{B}-n_{B}\right) \leq n_{A}$ and $m_{B}-n_{B} \leq$ $m_{A}-n_{A}$ : Here, $\mathrm{Tx}_{1}$ transmits $\left(n_{A}+n_{B}\right)$ symbols using sub-channels $A$ and $B$. Additionally, it transmits $\left(\left(m_{A}-\right.\right.$ $\left.\left.n_{A}\right)-\left(m_{B}-n_{B}\right)\right)$ symbols intended for $\mathrm{Rx}_{2}$ underneath the top $n_{A}$ levels in sub-channel $A$. Tx 2 sends $\left(m_{A}-n_{A}\right)$ symbols from the top and the remaining $\left(m_{B}-n_{B}\right)$ symbols underneath the top $\left(n_{A}-\left(m_{B}-n_{B}\right)\right)$ levels. The effective interference at each user is $\left(m_{B}-n_{B}\right)$ symbols which can be resolved in sub-channel $B$.

2) $m_{A}+m_{B}>2\left(n_{A}+n_{B}\right)$ : In this case, we are interested in achieving the rate pair:

$$
R_{1}=R_{2}=n_{A}+n_{B}
$$

To achieve this point, both $\mathrm{Tx}_{1}$ and $\mathrm{Tx}_{2}$ transmit on the top $n_{A}$ levels in sub-channel $A$ and top $n_{B}$ levels in subchannel $B$. Both transmitters remain silent on the bottom $n_{B}$ levels, and can transmit interference mitigation symbols in the remaining $m_{B}-2 n_{B}$ levels in sub-channel $B$. The interference at both receivers in sub-channel $A$ is $2 n_{A}-m_{A}$ bits, which can be mitigated in sub-channel $B$, since $m_{B}-$ $2 n_{B} \geq 2 n_{A}-m_{A}$.

As illustrative examples in Regime $2 a$, we consider two LDICs: the $(5,4,3,1)$-LDIC and the $(3,2,4,1)$-LDIC. Note that for the $(5,4,3,1)$-LDIC, $m_{A}+m_{B}=8 \leq 2\left(n_{A}+n_{B}\right)=10$. The rate pair we wish to achieve is $(5,3)$. For $(3,2,4,1)$-LDIC, $m_{A}+m_{B}=7>2\left(n_{A}+n_{B}\right)=6$. Therefore, the desired rate pair to achieve is $(3,3)$. We illustrate how the desired points are achieved in Fig. 10.

\section{COnClusions}

We characterized the capacity region of the two-user parallel LDIC completely, and in the process we showed that even symmetric parallel ICs are, in general, not separable. Further, we characterized the exact regions where the symmetric parallel LDIC is indeed separable. Interestingly, we observe that parallel LDICs are separable in a large area of interest within the "weak interference" regime.

\section{REFERENCES}

[1] C. E. Shannon. Two way communication channels. In 4th Berkeley Symposium on Mathematical Statistics and probability, volume 1, pages 611-644, Jul. 1961.

[2] R. Ahlswede. The capacity region of a channel with two senders and two receivers. The Annals of Probability, 2(5):805-814, Oct. 1974.

[3] A. B. Carleial. A case where interference does not reduce capacity. IEEE Transactions on Information Theory, 21(5):569-570, Sep. 1975.

[4] H. Sato. The capacity of the Gaussian interference channel under strong interference. IEEE Transactions on Information Theory, 27(6):786-788, Nov. 1981

[5] V. S. Annapureddy and V. V. Veeravalli. Gaussian interference networks: Sum capacity in the low-interference regime and new outer bounds on the capacity region. IEEE Transactions on Information Theory, 55(7):3032-3050, Jul. 2009.

[6] A. S. Motahari and A. K. Khandani. Capacity bounds for the Gaussian interference channel. IEEE Transactions on Information Theory, 55(2):620-643, Feb. 2009

[7] X. Shang, G. Kramer, and B. Chen. A new outer bound and the noisyinterference sum-rate capacity for Gaussian interference channels. IEEE Transactions on Information Theory, 55(2):689-699, Feb. 2009.

[8] T. S. Han and K. Kobayashi. A new achievable region for the interference channel. IEEE Transactions on Information Theory, 27(1):49-60, Jan. 1981.

[9] R. H. Etkin, D. Tse, and H. Wang. Gaussian interference channel capacity to within one bit. IEEE Transactions on Information Theory, 54(12):5534-5562, Dec. 2008.

[10] G. Bresler and D. Tse. The two user Gaussian interference channel: a deterministic view. European Transactions on Telecommunications, 19:333-354, Apr. 2008.

[11] M. H. M. Costa and A. A. El Gamal. The capacity region of a class of deterministic interference channels. IEEE Transactions on Information Theory, 28(2):343-346, Mar. 1982.

[12] A. S. Avestimehr, S. N. Diggavi, and D. Tse. Wireless network information flow: A deterministic approach. IEEE Transactions on Information Theory, 57(4):1872-1905, Apr. 2011.

[13] C. Suh and D. Tse. Feedback capacity of the Gaussian interference channel to within 2 bits. IEEE Transactions on Information Theory, 57(5):2667-2685, May 2011

[14] A. S. Avestimehr, A. Sezgin, and D. Tse. Capacity of the two way relay channel within a constant gap. European Transactions on Telecommunications, 21(4):363374, Jun. 2010.

[15] S. Saha and R. Berry. Symmetric k-user Gaussian interference channels: Approximate sum-capacity via deterministic modeling. In Annual Allerton Conference on Communication, Control, and Computing, Sep. 2012.

[16] L. Quoc, R. Tandon, M. Motani, and H. V. Poor. The capacity region of the linear deterministic interference channel with partial feedback. In Annual Allerton Conference on Communication, Control, and Computing, Oct. 2010

[17] S. M. Perlaza, R. Tandon, and H. V. Poor. The Nash equilibrium region of the linear deterministic interference channel with feedback. In Annual Allerton Conference on Communication, Control, and Computing, Oct. 2010

[18] S. Saha and R. Berry. The combinatorial structure of linear deterministic interference channels. In IEEE Information Theory Workshop, Sep. 2012.

[19] V. R. Cadambe and S. A. Jafar. Parallel Gaussian interference channels are not always separable. IEEE Transactions on Information Theory, 55(9):3983-3990, Sep. 2009.

[20] S. T. Chung and J. M. Cioffi. The capacity region of frequencyselective Gaussian interference channels under strong interference. IEEE Transactions on Communications, 55(9):1812-1821, Sep. 2007.

[21] C. Sung, K. W. K. Lui, K. W. Shum, and H. C. So. Sum capacity of one-sided parallel Gaussian interference channels. IEEE Transactions on Information Theory, 54(1):468-472, Jan. 2008.

[22] X. Shang, B. Chen, G. Kramer, and H. V. Poor. Noisy-interference sum-rate capacity of parallel Gaussian interference channels. IEEE Transactions on Information Theory, 57(1):210-226, Jan. 2011.

[23] L. Sankar, X. Shang, E. Erkip, and H. V. Poor. Ergodic fading interference channels: Sum-capacity and separability. IEEE Transactions on Information Theory, 57(5):2605-2626, May 2011.

[24] S. Saha and R. Berry. Parallel linear deterministic interference channels with feedback: Combinatorial structure and separability. In IEEE International Symposium on Information Theory, Jul. 2013. 